\title{
1 Improving range shift predictions: enhancing the power of traits
}

2 Running Title: Improved range shift predictions via traits.

3 Anthony F. Cannistra ${ }^{+1}$, Lauren B. Buckley ${ }^{1}$

4 1. Department of Biology, University of Washington, Seattle, WA 98195-1800, USA

5 +. Corresponding author: Anthony F. Cannistra, Phone: (401) 793-0916, Email:

6 tonycan@uw.edu, ORCID: 0000-0002-9852-6291

7 Abstract Accurately predicting species' range shifts in response to environmental change is a

8 central ecological objective and applied imperative. In synthetic analyses, traits emerge as

9 significant but weak predictors of species' range shifts across recent climate change. These

10 studies assume linearity in the relationship between a trait and its function, while detailed

11 empirical work often reveals unimodal relationships, thresholds, and other nonlinearities in many

12 trait-function relationships. We hypothesize that the use of linear modeling approaches fails to

13 capture these nonlinearities and therefore may be under-powering traits to predict range shifts.

14 We evaluate the predictive performance of four different machine learning approaches that can

15 capture nonlinear relationships (ridge-regularized linear regression, ridge-regularized kernel

16 regression, support vector regression, and random forests). We validate our models using four

17 multi-decadal range shift datasets in montane plants, montane small mammals, and marine fish.

18 We show that nonlinear approaches perform substantially better than least-squares linear

19 modeling in reproducing historical range shifts. In addition, using novel model observation and

20 interrogation techniques, the trait classes (e.g. dispersal- or diet-related traits) that we identify as

21 primary drivers of model predictions are consistent with expectations. However, disagreements 
22 among models in the directionality of trait predictors suggests limits to trait-based statistical

23 predictive frameworks.

24 Keywords: traits, range shifts, nonlinear modeling, ecological forecasting, global change

25 responses, distribution, vulnerability

\section{INTRODUCTION}

27 Species have been responding to recent climate change by tracking their environment in space or

28 time, adapting or acclimating, or facing declines (Parmesan, 2006), but we are largely unable to

29 predict how particular species will respond (Maguire et al., 2015). Extensive documentation of

30 shifts in distribution and seasonal timing (phenology) reveal that responses vary among species

31 markedly in direction and extent (Rapacciuolo et al., 2014). Detailed empirical studies often

32 succeed in identifying functional traits that govern climate change responses (e.g., Adrian et al.,

33 2006) and consequently functional ecology has been rapidly gaining prominence in climate

34 change ecology (Buckley \& Kingsolver, 2012). However, attempts to use traits to predict the

35 relative magnitude of responses among species generally identify traits that are significant, but

36 weak (accounting for $\sim 16 \%$ of the among species variation in range shifts, Buckley \&

37 Kingsolver, 2012), predictors of climate change responses (Estrada et al., 2016; MacLean \&

38 Beissinger, 2017). How can we close the discrepancy between traits predicting responses well in

39 detailed studies but poorly in broad studies? What statistical techniques will allow us to

40 generalize the importance of traits in mediating climate change responses?

41 Addressing such questions is imperative for anticipating and adapting to the biological

42 impacts of climate change. Indeed, traits are already being used to predict species' sensitivity to

43 climate change in vulnerability frameworks (Foden et al., 2013). However, the frameworks 
44 remain largely untested and perform poorly in tests (Wheatley et al., 2017). Most attempts to use

45 species' traits to predict the magnitude of their climate change responses rely on linear regression

46 (Buckley \& Kingsolver, 2012; MacLean \& Beissinger, 2017), yet detailed empirical studies

47 often reveal non-linear relationships between traits and their function (Stenseth \& Mysterud,

48 2002). Unimodal relationships and thresholds are common. For example, extreme diet

49 specialization may drive a species to track the range shift of a food item (Diamond et al., 2011),

50 but reducing diet specialization only slightly may alleviate the need for a species to track its

51 food. Diet generalization could facilitate species moving to capitalize on newly climatically

52 suitable habitat, yielding a unimodal relationship between diet specialization and the magnitude

53 of range shifts. Likewise, low dispersal ability may prevent a species from tracking its

54 environmental niche (Schloss et al., 2012), but the threshold of dispersal ability that allows

55 species to track their niche may be relatively low. Trade-offs among traits and differences in the

56 developmental dependencies of traits may also produce nonlinearities (Fitt et al., 2018). Can

57 statistical techniques that allow for non-linear relationships between traits and species' responses

$58 \quad$ improve our predictive ability?

Non-linear modelling techniques have been underutilized in predicting climate change

60 responses (Olden et al. 2008). Standard approaches to capture variable interactions and

61 nonlinearities in linear regressions (such as the explicit inclusion of interacting variables or

62 polynomial expansion) rely on prior knowledge or model selection techniques to determine

63 which variables to select. Other model types, such as machine learning approaches that optimize

64 model parameters, are better suited to capture functional relationships among variables. These

65 models, while offering statistical-robustness and efficiency, can be opaque and rarely afford clear 
66 coefficients to inspect when assessing the model's learned correlations. However, machine

67 learning developments offer new approaches for inspecting model performance and predictions.

68 Here we assess whether machine learning-based models can better use species' traits to

69 predict the magnitude and direction of range shifts observed in response to recent climate

70 change. First, we consider whether several models which are able to capture nonlinear

71 relationships can outperform linear models in their predictive ability. Second, we use recently

72 developed model interpretability techniques to ask whether model predictions are consistent

73 across modelling approaches and concur with ecological theory. Novel model inspection

74 approaches can reveal details of model predictions, which addresses reasonable concerns about

75 the "black box" nature of many machine learning-based models. We assess model performance

76 and robustness using four datasets encompassing a broad taxonomic range. The number of

77 included species ranges from 20 to 176 and range shifts were observed over time spans ranging

78 from 30 to $100+$ years. Each dataset was derived from previous evaluations of traits as range

79 shift predictors and consists of a list of focal species, associated species-level traits, and a range

80 shift metric. We examine (1) whether non-linear methods can improve predictive ability of traits

81 compared to linear methods, (2) whether the novel methods identify important traits consistent

82 with significant results from other studies, and (3) whether the directionality of the modeled

83 effect of traits is consistent across model types.

\section{MATERIALS AND METHODS}

85 We describe the nonlinear modeling approaches, a framework for interpreting model predictions,

86 and the assembly of range shift and trait data to determine whether traits can play a more

87 powerful role in predicting ecological responses to climate change. 
88 Modeling Approach We applied three classes of learning algorithms: regularized linear

89 regression, kernel-based regression, and tree-based regression. Regularization is a modification

90 to generalized linear regression that limits model complexity to avoid (Hastie et al., 2009).

91 Several types of regularization exist; we chose to use a "ridge"-regularized linear model, which

92 imposes a penalty on the magnitude of each learned coefficient. The cumulative effect of this

93 regularization procedure is a set of coefficients which both minimize prediction error on the

94 training data and prevent overfitting. These coefficients can be interpreted explicitly as with

95 ordinary least squares regression.

While regularization reduces overfitting when compared to a standard least-squares linear

97 fit, regularized linear models are still not able to capture nonlinearities among or interactions

98 between predictive variables. To remedy this, we employ two additional classes of models:

99 kernel-based regression and tree-based regression. A "kernel" is a function which projects a set

100 of input data, often into a high-dimensional space, to allow for the linear "separability" of the

101 data for the purposes of classification or regression (Hastie et al., 2009). The Kernel Ridge

102 method employed herein uses a radial basis function (or squared exponential) kernel applied to

103 the training data and fits a ridge-regularized linear model to this transformed input. As a result of

104 this transformation the learned coefficients, while regularized, are not immediately interpretable.

105 We also evaluate a kernel-based technique known as a support vector machine (SVM). This

106 popular learning method can be formulated for regression, is robust to outliers, and can capture

107 nonlinearities and variable interactions through a similar radial basis function kernel as in the

108 Kernel Ridge approach. Like the Kernel Ridge method, the SVM regressor does not have

109 interpretable coefficients. Finally, we train a random forest regression algorithm to evaluate the

110 performance of tree-based methods. All of these models are implemented in the Python 
111 programming language using the scikit-learn software package (Pedregosa et al., 2011), though

112 all analyses can be computed in the R language using available machine learning packages. All

113 code for this project is available on GitHub at https://github.com/huckleylab/cc_traits.

114 For comparison to the original analyses, we train an ordinary least squares regression

115 model, which assumes linear relationships and no variable interaction. While the original

116 analyses were mostly conducted in a single-variable framework (that is, to assess the effect size

117 of $M$ different potential predictive variables $M$ models were trained, each model containing only

1181 variable), we include all variables in single analyses to enable the models to capture variable

119 interactions and to follow a common predictive modeling paradigm. This multivariate approach

120 is a standard one in machine-learning based predictive analytics (Hastie et al., 2009).

1212.2 Evaluation We use data subsets for performance evaluation. To assess the predictive

122 performance of our models we employ a $k$-fold cross-validation scheme (Hastie et al. 2009)

123 combined with a squared error loss function. This cross-validation technique has been shown to

124 estimate expected prediction error (Hastie et al. 2009) by randomly partitioning data points into $k$

$125=10$ subsets, each with $N / k$ members; $k-1$ subsets are used to fit the model (the "training set"),

126 reserving one subset for testing model performance. Each of the $k$ subsets is used exactly once

127 for evaluation. A mean squared error (MSE) loss is then computed for the model prediction of

128 range shift magnitude in the reserved test data, which is the sum of squared differences between

129 predicted and actual range shift magnitude over all data in the testing set. This process is

130 repeated $k$ times and a mean statistic is computed across the $k$ MSE values that result (known as

131 a "cross-validation mean"). The units of MSE estimates are those of the range shift, so MSE

132 provides a direct assessment of model predictive ability. The same cross-validation mean

133 approach is used to compute average variable importance values to evaluate model drivers, 
134 described below. In these experiments we choose $k=10$, which is generally sufficient for robust 135 performance estimates (Hastie et al. 2009).

2.3 Model Interpretation The core of any basic regression analysis is typically an inspection of

137 the significance and direction of the coefficients of a fitted model. However, the kernel methods 138 employed herein (Kernel Ridge, SVM) do not expose any interpretable coefficients. To address 139 this, we utilize the Shapley additive feature value method, proposed in Lundberg \& Lee (2017) 140 and described in Supplementary Material 1. To compare the learning techniques, we use either 141 mean regression coefficients (for OLS and Ridge regression), mean Shapley variable importance 142 values (Kernel Ridge and SVM), or mean Gini variable importance values (Random Forest;

143 Breiman, 2001) to rank all variables such that each feature has an importance ranking for each of 144 the several regression methods. We use the sign of Shapley and coefficient values to compare the 145 directionality of predicted trait drivers for models other than random forests (Gini scores are an 146 unsigned information-theoretic measure). All means are $k$-fold cross-validated means.

$147 \quad$ 2.4 Trait and Range Shift Data We evaluate our approach independently by replicating 148 analysis across four datasets that (1) repeated historical surveys or conducted continuous surveys 149 along latitudinal or elevational gradients to quantify shifts in northern or upper elevation range 150 boundaries over at least three decades of change and (2) included all surveyed species (i.e. rather 151 than including only species that shifted significantly). The first two data sets are those used by 152 Angert et al. (2011) to assess the predictive power of traits. These datasets supplement trait data 153 with elevational range shift data for Swiss alpine plants (Holzinger et al., 2008; $N=139$ ) and for 154 Western North American small mammals (Moritz et al., 2008). A third database from Rumpf et 155 al. (2018) consists of elevational range shifts for European montane plants coupled with 156 empirically measured trait data supplemented with data from the TRY Plant Trait Database 
157 (Kattge et al., 2011; https://www.try-db.org and Supplementary Information, Section 2.2) and

158 other databases (e.g. Bjorkman et al., 2018). These two plant databases were chosen in an effort

159 to directly replicate data used in previous range shift studies, despite some overlap among them.

160 The fourth database was created by pairing estimates of latitudinal range shifts from coastal

161 North American marine fish surveys (Pinsky et al., 2013) with functional trait data in Fishbase

162 (https://www.fishbase.org, Froese \& Pauly, 2010 and Supplementary Information, Section 2.1).

163 Each dataset includes a directional range shift: negative values indicate shifts downward in

164 elevation $(\mathrm{m})$ for the first three datasets and equatorward in latitude (degrees) for the marine

165 dataset. We remove samples which are missing any traits, one-hot encode categorical traits (i.e.,

166 generate one boolean column for each category), and normalize/center the numeric traits to have

167 zero mean and unit norm. After this processing the Swiss plants dataset contains $N=20$ species

168 and $d=38$ traits (Table $\mathrm{S} 1$ ); the Yosemite mammal dataset contains $N=28$ species and $d=19$

169 traits (Table S2); the European plants dataset contains $N=176$ species and $d=18$ traits (Table

$170 \mathrm{~S} 3$ ); the marine fish dataset contains $N=76$ species and $d=17$ traits (Table S4).

\section{RESULTS}

172 We find that the machine learning approaches improve predictive performance over an ordinary

173 least squares (OLS) model baseline. For two datasets (Swiss alpine plants and Western NA

174 mammals), the four machine learning approaches perform substantially better than the OLS

175 models but similarly to each other. For the other two datasets (European montane plants and

176 marine fish), the performance advantages of the machine learning approaches are less substantial

177 and the four machine learning approaches differ more in their performance. Support Vector

178 Regression (SVR) and Kernel Ridge emerge as the most performant methods across datasets

179 (Figure 1), reducing mean error in range shift estimates by an average of $62.8 \%$ and $61.6 \%$ 
relative to OLS, respectively (medians of $50.6 \%$ and $49.6 \%$, respectively). We focus on results

181 from the Swiss alpine plants dataset (Angert et al., 2011; Holzinger et al., 2008) to demonstrate

182 findings. The initial OLS analysis found that individual predictors accounted for relatively little

183

variance in the extent of the plants' elevational range shifts $\left(\mathrm{R}^{2}=0.05-0.18\right.$, Angert et al., 2011)

In addition to reducing MSE, the traits found to be important predictors in machine learning models correspond to those identified in previous analyses and ecological theory. The most performant models for these data (SVR and Kernel Ridge, lowest MSE: Figure 1a) identify dispersal-related traits (e.g., the timing, height, and duration of seed shed along with seed size and dispersal mode for the Alpine and European Plants) as the most important variables in predicting range shift magnitude (Figure 2a), which is consistent with our expectation and prior work (Angert et al. 2011). In the original analysis of the European Plants data (Rumpf et al., 2018) the indicator of thermal adaptation (cool to warm adaptation, "TemperatureIndicator") was a primary predictor of range shifts; our models selected the same variable as most important (Figure 2b). The previous Western NA mammal analysis (Angert et al. 2011) identified altitudinal limit as a significant predictor and longevity as a relatively strong, but non-significant, predictor. Our models select those two variables as the most important predictors (Figure 2c).

Both the initial surveys (Pinsky et al., 2013) and our analysis failed to identify strong trait predictors of marine range shifts and non-linear methods yielded less improvement of MSE than the other datasets. However, habitat traits such as whether species are pelagic are top predictors consistent with compilations of individual studies (Poloczanska et al., 2013).

The models employed in this analysis also demonstrate cross-model consistency in identifying trait drivers of range shifts (Figure 2). In other words, separate models tend to agree on trait rank, especially in the top 5 traits, suggesting a common effect despite significantly 
different modeling methodologies (and evaluation strategies). However, there tends to be more agreement among machine learning models than between OLS and machine learning models. In particular, some traits for which thresholds seem likely are less important predictors in OLS than in machine learning models: higher seed shed heights and longer seed shed durations in alpine plants may not lead to more dispersal once thresholds are reached (Figure 2a). example, all models for European plants (except Kernel Ridge) suggest that thermophilic species

212 (except SVR) find that mammals with greater longevity and higher altitudinal limits exhibit

213 smaller altitudinal range shifts. In both these examples, the exceptional model suggests the

214 opposite relationship for both traits. Agreement is sometimes strongest within regression (OLS,

215 Ridge) and machine learning (Kernel Ridge, SVR) type models. For example, the machine

216 learning models suggest alpine plants with higher seed shed shift their distribution further,

217 contrary to the findings of regression models. However, the mixed results suggest limits to the

218 predictive capacity of species' traits (see Discussion). It is important to note that these measures

219 of variable contribution are computed in distinct ways depending on the modeling methodology,

220 and it remains to be seen whether these methods of variable importance are suitable to determine

221 effect directionality. In addition, using these heterogeneous metrics to compare effect

222 directionality must be done with caution.

\section{DISCUSSION}

224 We find that non-linear modeling methods enhance the ability of traits to accurately predict

225 observed range shifts. Our findings match biological intuition that biological processes, which 
respond to environmental conditions and are mediated by species' traits, are rarely linear

227 (Stenseth \& Mysterud, 2002). Importantly, we have shown that the novel statistical models

228 maintain biological rigor by identifying similar predictor traits. However, some disagreements in

229 the directionality of the relationship between trait values and range shift magnitude suggest

230 limits to trait-based statistical prediction frameworks.

232 predictions, such as analyses of climate change vulnerability. The substantially better predictive

233 performance of non-linear models relative to linear models suggests vulnerability analyses

234 frameworks based on species' traits (e.g., Foden et al., 2013; Pacifici et al., 2017) should be

235 adapted to account for non-linearities. However, disagreements in the directionality of trait

236 predictors and the variability of performance improvement suggest that even non-linear methods

237 for relating traits to climate change responses may have limited predictive accuracy. More

238 mechanistic approaches that describe the processes by which traits mediate fitness and

239 demographic responses to the environment may be required for predictions that require high

240 levels of accuracy (Buckley \& Kingsolver, 2012; Urban et al., 2016). At a minimum, these

241 mechanistic approaches will be useful for refining non-linear methodologies for using traits to

242 predict climate change responses.

Models that allow for non-linearities should be employed to further reevaluate

244 expectations for how traits govern range shifts. A meta-analysis across range shift studies found

245 at best moderate support for dispersal ability (body size: 22\%, migratory strategy: 10\%,

246 movement ability: 50\% of studies uphold predicted relationship), reproductive potential

247 (fecundity: 36\%, longevity: 60\%) and ecological generalization (diet breadth: 27\%, habitat

248 breadth: 43\%) as predictors of range shift magnitude (MacLean \& Beissinger, 2017). The large 
249 gap between expectations and observations highlights the need for novel predictive methods.

250 Translating spatial range shifts into metrics of environmental niche tracking (e.g., velocity of

251 climate change, Loarie et al., 2009) may also enhance predictive capacity.

253 predictor traits. The availability of trait data has increased substantially since some of our

254 datasets were compiled (e.g., Angert et. al 2011), so refining traits may improve predictive

255 capacity. Still, needs for additional trait data addressing issues such as physiology and

256 evolutionary potential are substantial and will likely require concerted data collection efforts

257 (Urban et al., 2016). Since species' traits are likely to be phylogenetically conserved,

258 phylogenetic signal in range shifts can be used to assess the potential to use traits to predict range

259 shifts. High phylogenetic signal but weak predictive performance of traits would suggest that

260 improving the traits used as predictors can enhance predictive capacity. The initial analyses (e.g.,

261 Angert et. al 2011) that accounted for phylogeny found limited phylogenetic signal in range

262 shifts. We did not account for phylogeny because it is not straightforward to do so in the machine

263 learning models. A recent synthesis of range shift studies (Diamond, 2018) found variable but

264 generally weaker phylogenetic signal in range shifts than in physiological, morphological, and

265 life-history traits. The finding indicates limits to the predictive capacity of traits.

267 model interrogation approaches - has been reliably employed across many predictive contexts

268 but has yet to be fully embraced by ecologists. The emerging wealth of publicly-available

269 ecological and environmental data, combined with the pressing need for reliable ecological

270 forecasts that are useful in decision-making frameworks, makes this flexible and data-intensive

271 approach a natural fit. Despite promising results, our approach presents several challenges to 
272 adoption. Of particular relevance to the ecology community is the lack of traditional statistical

273 techniques to evaluate these methods. Following the machine learning community, we employ $k$ -

274 fold cross-validation to lend statistical robustness to the pertinent evaluative criteria for our

275 models (here, mean squared error). In addition, the use of recent advances in model inspection

276 methods (Shapley values from Lundberg \& Lee, 2017) represents a necessary departure from the

277 manual inspection and testing of linear model coefficients. As the field of model interpretation

278 grows, ecologists can leverage these developments to verify the ecological processes

279 underpinning the predictions of these unconventional modeling approaches. Understanding and

280 acknowledging these shifts in method evaluation and inspection approaches are critical steps to

281 leveraging these more performant statistical modeling approaches in the ecology community.

283 additional taxa, a greater number of species, and using novel model interrogation techniques. In

284 addition, the approach has the potential to improve predictive accuracy in other ecological

285 domains relevant to policy and decisions making (species distribution modeling, forecasting of

286 ecological carbon flux, etc.). However, as is demonstrated by the range of predictive

287 performance improvement across datasets (e.g. between the Alpine Plants and the European

288 Plants datasets, Figure 1), care must be taken to evaluate any novel modeling result

289 conservatively and through a lens of data quality and available latitude for improvement.

290 ACKNOWLEDGEMENTS We thank contributors to TRY, Fishbase, and the other datasets

291 and input and assistance from Amy Angert, Malin Pinsky, Ray Huey, and particularly Sabine

292 Rumpf. This work was supported by the National Science Foundation [IGERT DGE-1258485

293 fellowship to A.F.C., a Graduate Research Fellowship to A.F.C, and DBI-1349865 to L.B.B.].

\section{References}


295

296

297

298

299

300

301

302

303

304

305

306

307

308

309

310

311

312

313

314

315

Adrian, R., Wilhelm, S., \& Gerten, D. (2006). Life-history traits of lake plankton species may govern their phenological response to climate warming. Global Change Biology, 12(4), $652-661$.

Altermatt, F. (2010). Tell me what you eat and I'll tell you when you fly: diet can predict phenological changes in response to climate change. Ecology Letters, 13(12), 1475-1484.

Angert, A. L., Crozier, L. G., Rissler, L. J., Gilman, S. E., Tewksbury, J. J., \& Chunco, A. J. (2011). Do species' traits predict recent shifts at expanding range edges? Ecology Letters, $14(7), 677-689$.

Bjorkman, A. D., Myers-Smith, I. H., Elmendorf, S. C., Normand, S., Thomas, H. J. D., Alatalo, J. M., .. Zamin, T. (2018). Tundra Trait Team: A database of plant traits spanning the tundra biome. Global Ecology and Biogeography, 27(12), 1402-1411.

Breiman, L. (2001). Random Forests. Machine Learning, 45(1), 5-32.

Buckley, L. B., \& Kingsolver, J. G. (2012). Functional and Phylogenetic Approaches to Forecasting Species’ Responses to Climate Change. Annual Review of Ecology, Evolution, and Systematics, 43(1), 205-226.

Diamond, S. E., Frame, A. M., Martin, R. A., \& Buckley, L. B. (2011). Species’ traits predict phenological responses to climate change in butterflies. Ecology, 92(5), 1005-1012.

Estrada, A., Morales-Castilla, I., Caplat, P., \& Early, R. (2016). Usefulness of Species Traits in Predicting Range Shifts. Trends in Ecology \& Evolution, 31(3), 190-203.

Fitt, R. N. L., Palmer, S., Hand, C., Travis, J. M. J., \& Lancaster, L. T. (2018). Towards an interactive, process-based approach to understanding range shifts: developmental and 
environmental dependencies matter. Ecography, $0(0)$.

317 Foden, W. B., Butchart, S. H. M., Stuart, S. N., Vié, J.-C., Akçakaya, H. R., Angulo, A., ...

318 Mace, G. M. (2013). Identifying the World's Most Climate Change Vulnerable Species: A 319 Systematic Trait-Based Assessment of all Birds, Amphibians and Corals. PLOS ONE, 8(6), $320 \quad 1-13$.

321 Froese, R., \& Pauly, D. (2010). FishBase.

322 Hastie, T., Tibshirani, R., \& Friedman, J. (2009). The Elements of Statistical Learning. In The Mathematical Intelligencer.

324 Holzinger, B., Hülber, K., Camenisch, M., \& Grabherr, G. (2008). Changes in plant species 325 richness over the last century in the eastern Swiss Alps: elevational gradient, bedrock 326 effects and migration rates. Plant Ecology, 195(2), 179-196.

327 Kattge, J., Díaz, S., Lavorel, S., Prentice, I. C., Leadley, P., Bönisch, G., ... Wirth, C. (2011). TRY - a global database of plant traits. Global Change Biology, 17(9), 2905-2935.

Lundberg, S. M., \& Lee, S.-I. (2017). A Unified Approach to Interpreting Model Predictions. In

MacLean, S. A., \& Beissinger, S. R. (2017). Species' traits as predictors of range shifts under contemporary climate change: A review and meta-analysis. Global Change Biology, 23(10), (Eds.), Advances in Neural Information Processing Systems 30 (pp. 4765-4774).

335 Maguire, K. C., Nieto-Lugilde, D., Fitzpatrick, M. C., Williams, J. W., \& Blois, J. L. (2015). 336 Modeling Species and Community Responses to Past, Present, and Future Episodes of 
Climatic and Ecological Change. Annual Review of Ecology, Evolution, and Systematics, $46(1), 343-368$.

Moritz, C., Patton, J. L., Conroy, C. J., Parra, J. L., White, G. C., \& Beissinger, S. R. (2008). Impact of a Century of Climate Change on Small-Mammal Communities in Yosemite National Park, USA. Science, 322(5899), 261-264.

Pacifici, M., Visconti, P., Butchart, S. H. M., Watson, J. E. M., Cassola, F. M., \& Rondinini, C. (2017). Species' traits influenced their response to recent climate change. Nature Climate Change, 7(3), 205-208.

Parmesan, C. (2006). Ecological and Evolutionary Responses to Recent Climate Change. Annual Review of Ecology, Evolution, and Systematics, 37(1), 637-669.

Pedregosa, F., Varoquaux, G., Gramfort, A., Michel, V., Thirion, B., Grisel, O., ... Duchesnay, E. (2011). Scikit-learn: Machine Learning in Python. Journal of Machine Learning Research, 12, 2825-2830.

Pinsky, M. L., Worm, B., Fogarty, M. J., Sarmiento, J. L., \& Levin, S. A. (2013). Marine Taxa Track Local Climate Velocities. Science, 341(6151), 1239-1242.

Poloczanska, E. S., Brown, C. J., Sydeman, W. J., Kiessling, W., Schoeman, D. S., Moore, P. J., ... Richardson, A. J. (2013). Global imprint of climate change on marine life. Nature Climate Change, 3, 919.

Rapacciuolo, G., Maher, S. P., Schneider, A. C., Hammond, T. T., Jabis, M. D., Walsh, R. E., ... Beissinger, S. R. (2014). Beyond a warming fingerprint: individualistic biogeographic responses to heterogeneous climate change in California. Global Change Biology, 20(9), 
2841-2855.

Rumpf, S. B., Hülber, K., Klonner, G., Moser, D., Schütz, M., Wessely, J., ... Dullinger, S. (2018). Range dynamics of mountain plants decrease with elevation. Proceedings of the National Academy of Sciences, 115(8), 1848-1853.

362

Schloss, C. A., Nuñez, T. A., \& Lawler, J. J. (2012). Dispersal will limit ability of mammals to track climate change in the Western Hemisphere. Proceedings of the National Academy of Sciences, $109(22), 8606-8611$.

Stenseth, N. C., \& Mysterud, A. (2002). Climate, changing phenology, and other life history traits: Nonlinearity and match-mismatch to the environment. Proceedings of the National

Urban, M. C., Bocedi, G., Hendry, A. P., Mihoub, J.-B., Pe’er, G., Singer, A., .. Travis, J. M. J. (2016). Improving the forecast for biodiversity under climate change. Science, 353(6304).

Wheatley, C. J., Beale, C. M., Bradbury, R. B., Pearce-Higgins, J. W., Critchlow, R., \& Thomas, C. D. (2017). Climate change vulnerability for species-Assessing the assessments. Global Academy of Sciences, 99(21), 13379-13381.

\section{$373 \quad$ Figure Legends}

374 Figure 1: The machine learning approaches reduce the mean squared error (MSE, 10-fold cross-

375 validation) of range shift predictions below the MSE of the standard linear regression approach

376 (OLS: ordinary least squares) across all three of four datasets (A, C, D). Box represents

377 interquartile range (lower to upper), central lines shows data median, and whiskers represent the 378 range of the data. Support Vector Regression (SVR) and Kernel Ridge models exhibit stronger 
379 performance than ridge regularized linear (Ridge) or Random Forest (RF) models across the

380 datasets. The MSE units correspond to the range shift metric (A-C: m, D:degrees latitude) and

381 thus directly indicate model performance.

382 Figure 2: The model approaches select similar traits as important (larger and red = more

383 important) for predicting range shifts across datasets. Traits are listed in order of decreasing

384 mean importance (increasing mean rank, right column) across all methods (except OLS) for each

385 dataset (panels). Model abbreviations are as in Figure 1.

386 Figure 3: Models tend to agree on the variable importance values and directionality of top-

387 ranking traits. We depict model coefficients (OLS, Ridge), Shapley feature importance values

388 (Kernel Ridge, SVR), or Gini feature importance scores (RF) for top ten traits by rank (Figure

$389[$ ranks]) for each dataset (rows). Model abbreviations are as in Figure 1 and traits are as in Figure

3902.

$391 \quad$ Figures

392

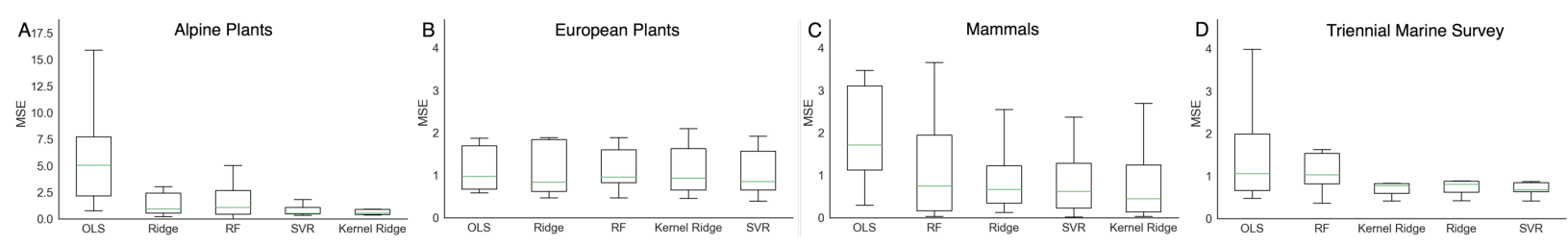

393 Figure 1 
bioRxiv preprint doi: https://doi.org/10.1101/2021.02.15.431292; this version posted February 16, 2021. The copyright holder for this preprint (which was not certified by peer review) is the author/funder. All rights reserved. No reuse allowed without permission.

A - Alpine Plants

Trait
Seed Shed Month (earliest)

\section{B - European Plants}

\begin{tabular}{|c|c|c|c|c|c|c|}
\hline Trait & OLS & RF & Ridge & Kernel Ridge & SVR & Mean \\
\hline Temperaturelndicator & - & - & $\bullet$ & 0 & - & $\bullet$ \\
\hline SeedReleaseHeight & $\bullet$ & - & $\bullet$ & 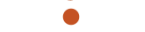 & - & $\bullet$ \\
\hline RelativeAbundance & - & O & - & - & $\bullet$ & $\bullet$ \\
\hline LifeSpan & - & 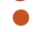 & $\bullet$ & - & $\bullet$ & - \\
\hline LifeStrategy_crs & $\bullet$ & - & 0 & 0 & $\bullet$ & - \\
\hline NoOfVegOffspings & - & $\bullet$ & - & $\bullet$ & 0 & - \\
\hline NutrientIndicator & - & - & - & - & 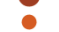 & - \\
\hline RetInFurSheep & - & - & - & $\bullet$ & ○ & - \\
\hline TerminalVelocity & - & - & - & - & - & - \\
\hline RetInFurCattle & - & - & - & - & - & - \\
\hline
\end{tabular}

C - Mammals

Trait

Altitudinal Limit

Longevity (years)

Bio1_std

Range Size (km2)

Facultative Hibernation (binary)

Rhythm: Diurnal + Nocturnal (binar.

Litter Size (individuals)

Granivore (binary)??

Rhythm: Diurnal (binary)

OLS

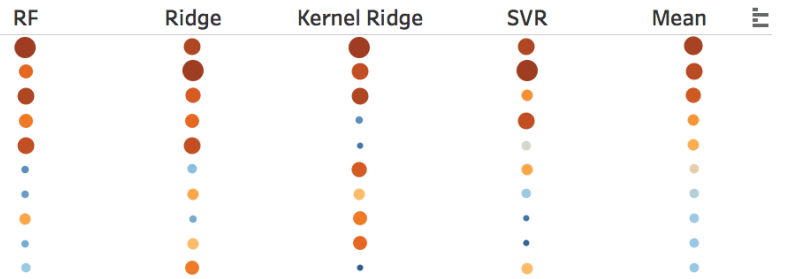

D - Triennial Marine Survey

Trait

DemersPelag_reef-associated

Length

DepthRangeDeep

DemersPelag_benthopelagic

Vulnerability

DemersPelag_demersal

Fresh_-1

DemersPelag_pelagic-oceanic

DemersPelag_bathydemersal
DemersPelag_pelagic-neritic

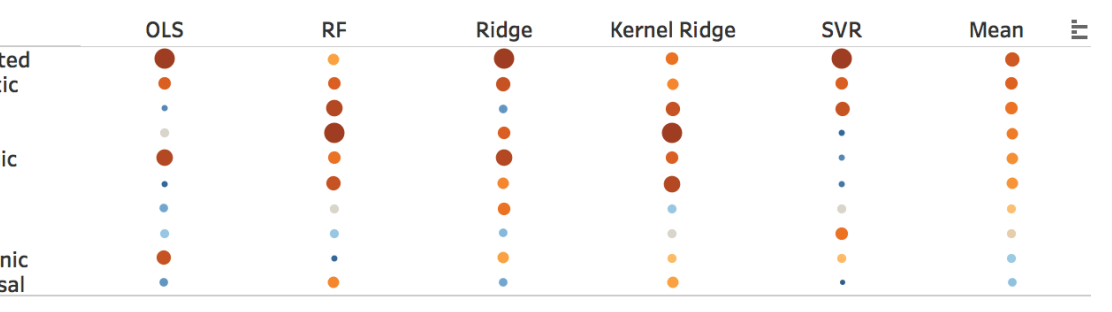

Low Rank - More Important

High Rank - Less Important

\section{$395 \quad$ Figure 2}


bioRxiv preprint doi: https://doi.org/10.1101/2021.02.15.431292; this version posted February 16, 2021. The copyright holder for this preprint (which was not certified by peer review) is the author/funder. All rights reserved. No reuse allowed without permission.
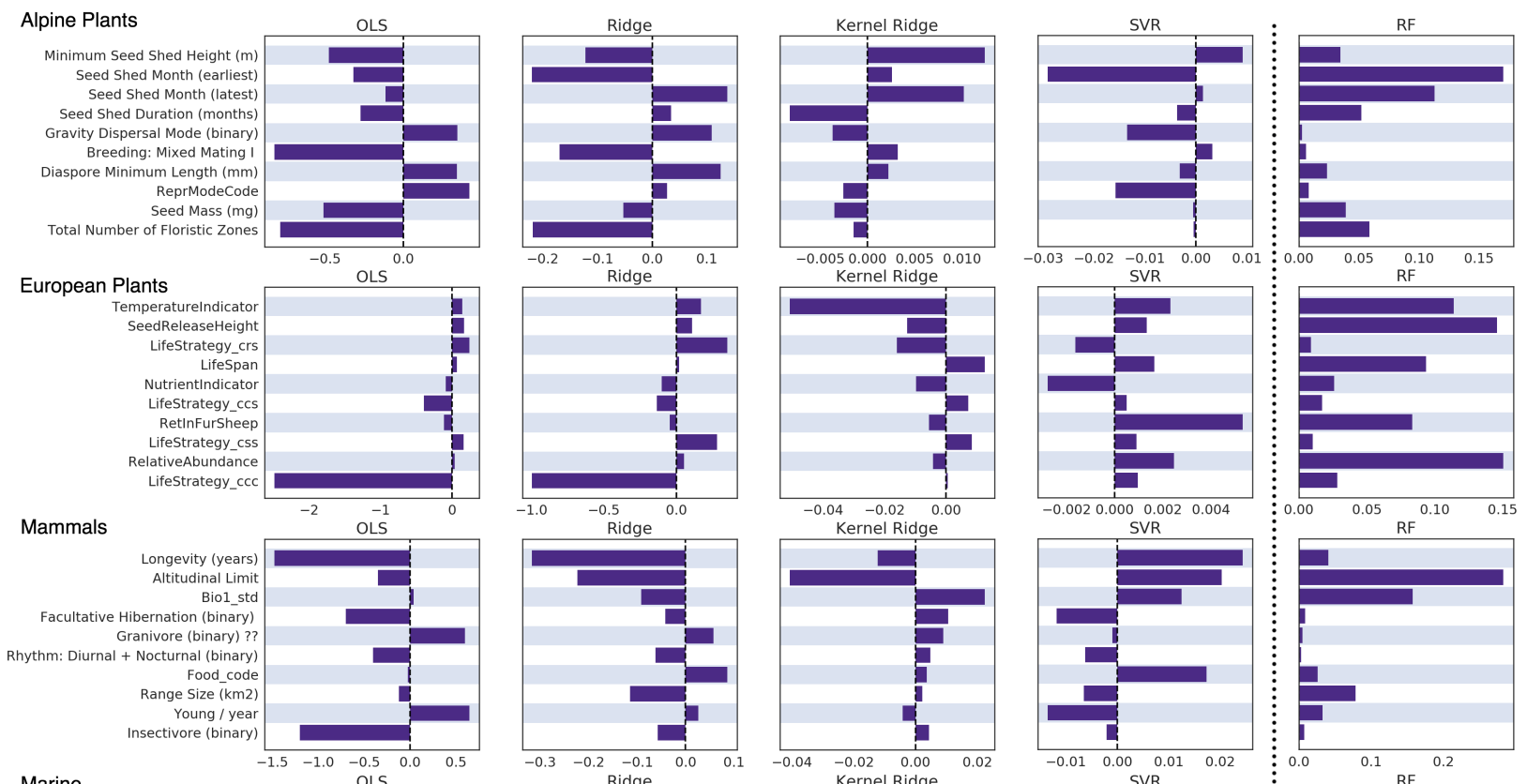

Marine
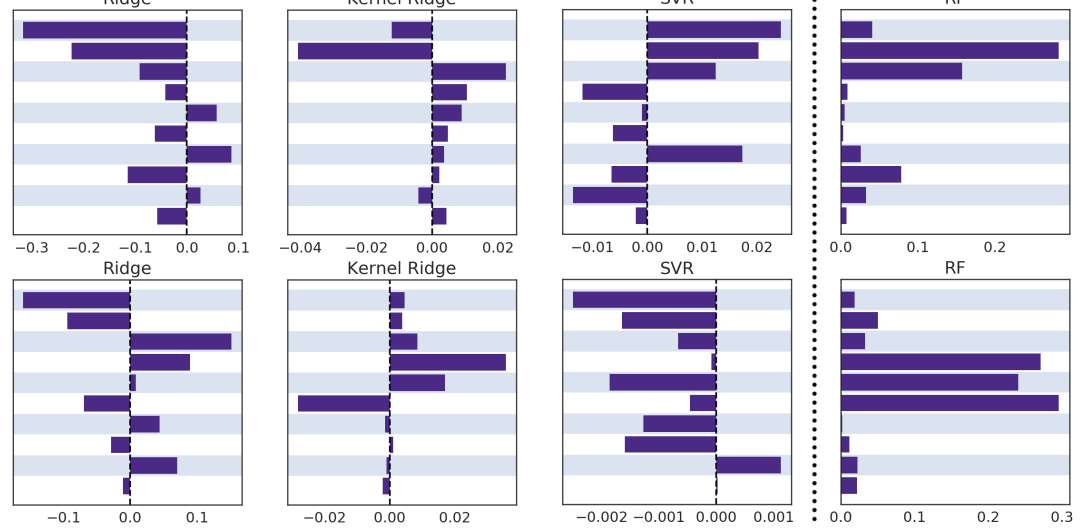

DemersPelag_reef-associated
DemersPelag_pelagic-neritic DemersPelag_pelagic-neritic
DemersPelag_benthopelagic DemersPelag_benthopelagic
DepthRangeDeep

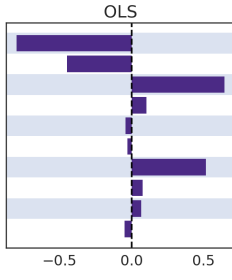

DemersPelag_pelagic-oceanic

DemersPelag_demersal

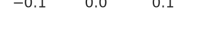

Variable Importance

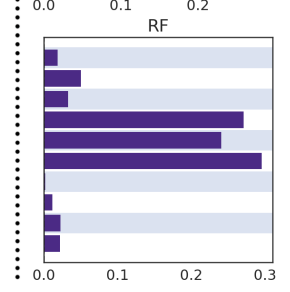

\section{$398 \quad$ Figure 3}

